\title{
Les activités de contribution dans la politique intranet d'un Conseil Général : de l'injonction à une sémio-politique de la « transversalité» en organisation
}

The Distributed Activity of Publishing within the Intranet Policy of a French Local Authority: From a Prescriptive Praxis in Institutions to a Semio-politic of Transversality in Organizations

Maryse Carmes

\section{OpenEdition}

Journals

Édition électronique

URL : http://journals.openedition.org/edc/1105

DOI : 10.4000/edc. 1105

ISSN : 2101-0366

Éditeur

Université Lille-3

Édition imprimée

Date de publication : 1 décembre 2009

Pagination : $19-40$

ISSN : 1270-6841

Référence électronique

Maryse Carmes, « Les activités de contribution dans la politique intranet d'un Conseil Général : de l'injonction à une sémio-politique de la «transversalité» en organisation», Études de communication [En ligne], 33 | 2009, mis en ligne le 01 décembre 2011, consulté le 01 mai 2019. URL : http:// journals.openedition.org/edc/1105; DOI : 10.4000/edc.1105

Ce document a été généré automatiquement le 1 mai 2019.

(ㄷ) Tous droits réservés 


\section{Les activités de contribution dans la politique intranet d'un Conseil Général : de l'injonction à une sémio-politique de la « transversalité» en organisation}

The Distributed Activity of Publishing within the Intranet Policy of a French Local Authority: From a Prescriptive Praxis in Institutions to a Semio-politic of Transversality in Organizations

Maryse Carmes

\section{Introduction}

1 A partir des pratiques professionnelles et de certaines narrations portant sur les TIC, cet article interroge les activités de contribution sur intranet en procédant à l'examen d'un rapport complexe entre une injonction institutionnelle et ses modes d'incarnation au sein d'une organisation. Les collectivités territoriales constituent ici notre terrain de recherche. Ce terrain est caractérisé par un puissant dispositif instituant une forme organisationnelle, redéfinie à l'aune de la notion de "transversalité ». Celle-ci est supposée exprimer la tendance modernisatrice des administrations, leur volonté d'améliorer leur performance, tendance et volonté s'appuyant sur une évolution spécifique des ingénieries intranets, une forme de management des processus infocommunicationnels et des processus coopératifs-collaboratifs. L'idée de transversalité est suffisamment vague pour permettre de multiples interprétations et recouvrir des expérimentations socio-techniques, techno-politiques, très diverses. Une opportunité s'offre donc pour relancer les enquêtes à partir de terrains spécifiques où se négocient, du niveau directionnel jusqu'au niveau des micro-pratiques, ses modalités d'application. A 
cet égard, dans les collectivités territoriales, la mise en œuvre de réseaux de contributeurs (catégorie spécifique de salariés-intranautes) permet d'observer, de manière peut-être modeste mais solide, les façons dont l'activité de contribution se trouve être, à la fois, aux prises avec l'intranet et avec l'injonction à la transversalité, ou du moins, avec la dimension enchantée de ce «maître-mot». Prendre la mesure de la complexité du couplage socio-technique qui s'opère ici, implique de rendre compte d'une pluralité d'actants et de l'existence de processus spécifiques. En premier lieu, nous présentons notre proposition d'analyse du couplage TIC-Transversalité en tant que script de l'e-organisation, et décrivons synthétiquement le rôle d'instances impliquées dans la fondation d'un modèle d'e-administration. Puis, nous analysons les résultats d'une recherche menée au sein d'un Conseil Général, en portant un regard critique sur la manière dont il se réapproprie ce script à partir de la mise en œuvre de son réseau de contributeurs. Ce travail s'appuie sur des entretiens individuels (agents et acteurs décideurs), des observations de pratiques, et enfin, sur une enquête en ligne réalisée en mai-juin 2008. Traversé de diverses tensions, on voit ainsi comment le projet intranet, supportant le mot d'ordre "transversalité ", s'accompagne d'une sémio-politique de l'innovation organisationnelle.

\section{Etudier la construction des scripts de l'e-organisation}

\section{Le script comme actant des politiques et pratiques organisationnelles}

2 Depuis le début des années 2000, l'utilisation de l'expression «e-organisation » par les professionnels témoigne de l'ancrage des TIC, et particulièrement des intranets, au sein des nouvelles politiques organisationnelles. L'e-organisation exprimerait l'adossement des processus info-communicationnels, des processus de travail, de leur management, aux technologies et systèmes d'information convergeant ainsi au sein du dispositif hétérogène que constitue l'intranet. La fondation en cours de modèles organisationnels est alors propice à la production collective de nombre de prescriptions, de scénarii d'excellence, de mots d'ordre. Dans un premier sens, nous définissons en tant que "script», l'actant opérant comme grille décisionnelle et comme instance de légitimation des politiques managériales. Ainsi, les scripts institués s'incarnent dans les injonctions et se déplacent via tout un ensemble de prescripteurs : consultants, associations professionnelles, responsables projets, institutions, etc. Même si ce versant sera particulièrement étudié dans cet article, nous devons préciser que les narrations, sous leur forme discursive, ne constituent qu'une des formes d'actants de l'agencement sur lequel repose la construction des «modèles » et donc des scripts eux-mêmes. En effet, il s'agit de prendre la mesure de l'enchevêtrement, sur des niveaux d'échelle variés, d'une multiplicité d'objets, d'agents, de réseaux, qui vont ainsi présider à la formation/ transformation des couplages socio-techniques. Dans un des textes fondateurs de la sociologie de la traduction, Michel Callon et Bruno Latour rappelaient à ce titre, la nécessité d'un dépassement des dichotomies social/technique, humain/non humain, micro/macro, pour pouvoir étudier «les stratégies qui enrôlent des corps, des discours, des sentiments, des lois, des organisations...» (Callon, Latour, 1981). Ainsi, le formatage des politiques organisationnelles s'effectue à partir d'un dispositif distribué où opèrent des objets techniques (les scripts d'usages s'incarnent dans les objets, leurs programmes, 
leurs interfaces), des règles, des expérimentations locales, des pratiques d'intranautes, des coalitions d'acteurs, des instances institutionnelles etc. Sans en préciser ici plus avant les modes d'expression et les modes opératoires (Carmes, 2008-b), le script résulte donc d'une agrégation, d'une combinatoire de caractéristiques techniques, de pratiques différenciées, de prescriptions, de stratégies, de contraintes normatives voire coercitives, et de doxas, qui se distribuent au sein des agencements par lesquels ils adviennent. Parmi les processus à l'oeuvre, on va pouvoir identifier des agents et des collectifs agissant, un certain temps, comme autant d'intercesseurs favorisant les "connexions" au sein de l'agencement et la réplication des décisions de chefs de projets. Pour ce qui est des collectivités territoriales, deux instances se distinguent particulièrement : les associations professionnelles et les institutions gouvernementales. Loin d'en déterminer a priori la puissance, la construction institutionnelle s'appuie sur un réseau d'acteurs et d'organisations multiples et se présente comme un véhicule parmi d'autres des scripts de l'e-organisation. Le "macro » nous dit Latour, «n'est ni au-dessus ni en-dessous des interactions : il vient s'ajouter à elles comme une autre connexion qui les alimente et s'en nourrit » (Latour, 2006, p 259). Les injonctions formulées ne sont donc pas exclusivement posées « extérieurement » aux acteurs-projets, aux dispositifs technologiques, car, pour partie, elles émergent et s'actualisent à partir des organisations elles-mêmes, se confrontent aux projets locaux et aux activités des salariés-intranautes. Le processus d'adoption et la mise en pratique de tel ou tel script ne peut alors s'envisager qu'au regard des puissances, des rapports de force s'établissant entre un certain nombre d'acteurs de l'agencement, ceux-ci pouvant alors avoir plus ou moins la liberté de souscrire ou pas aux divers scripts circulants et de les adapter.

\section{L'affirmation du script TIC-Transversalité}

3 A bien des égards, le rapport réciproque posé entre l'intranet et la transversalité tendrait à incarner les nouveaux modèles organisationnels, qui se développent sur fond d'une prescription, déjà bien établie au niveau des entreprises privées, en matière « d'intranet collaboratif». Les dispositifs événementiels, «les prix intranet» et les colloques organisés par diverses associations professionnelles ainsi que par la presse spécialisée, ont nourri un impératif de rupture vis-à-vis des intranets dits de "première génération ", massivement caractérisés par des logiques structuro-normatives et des politiques communicationnelles centralisées. Les responsables intranet se sont tournés vers des solutions coopératives/collaboratives bénéficiant pour la plupart d'une longue histoire, et aujourd'hui, les modèles en cours de construction s'établissent à nouveau à partir du repère-étalon que constitue internet et le «web 2.0 ». Ainsi, de manière récente, sont annoncés un autre modèle organisationnel et d'autres pratiques collectives de travail s'adossant à des projets qualifiés «d'intranets 2.0 ». Les scripts ici formalisés fondent l'idée d'une organisation redéfinie, à l'aune de l'idée générale de transversalité, maîtremot renvoyant à des exigences de coopération collective, de partage des biens informationnels et des savoirs, de socialisation numérique et augmentée (le social software faisant son apparition récente). Les nouvelles politiques rattachées aux activités de contributions en organisation se présentent donc comme une des expressions / traductions de ces ingénieries info-communicationnelles, qui tendraient à imposer l'idée d'un salarié-contributeur devenu un acteur direct des transformations de l'entreprise, un acteur de la transversalité. 


\section{Les prescriptions et les injonctions portées par des instances instituantes}

\section{Le constat d'un retard et l'alerte des associations professionnelles}

4 Dans l'univers des collectivités territoriales, modernisation, e-administration, transversalité etc. sont autant de mots d'ordre, de "valeurs", qui nourrissent les mêmes désirs d'adoption de certaines stratégies, les désirs de mises en conformité avec un « référentiel étalon ». Mais, l'adoption des scripts et leur réplication ont un préalable : la construction d'un consensus sur les problématiques dominantes et sur les actions correctives à mettre en oeuvre. En matière de politiques TIC, le diagnostic d'un retard des organisations territoriales (Conseils Régionaux, Conseils Généraux, Municipalités) est largement partagé. En 2007, des élus rappelaient « l'immaturité » et les risques de décrochages des pouvoirs publics. Ce constat est confirmé par différentes études. Selon l'enquête 2005 Localtis-Caisse des Dépôts et Consignations, 77\% des répondants (grandes collectivités en majeur) possédaient un intranet dont l'ancienneté serait de moins de 3 ans pour $64 \%$ des organisations interrogées (alors que pour près des deux tiers des grandes organisations privées la première version des intranets serait antérieure à 2002). Néanmoins, il serait erroné de considérer que les administrations françaises n'ont été sensibilisées aux TIC que bien après les entreprises privées. En effet, les associations professionnelles, issues spécifiquement du monde des collectivités territoriales, ont également joué un rôle d'alerte quant à la nécessité de penser l'enjeu des TIC. Une analyse de l'activité associative a montré le tissage d'un réseau très riche d'acteurs qui, à partir de la fin des années 90 , constitue une force d'influence non négligeable. Par exemple, les élus et les chefs de projets TIC qui ont fondé, en 2000, l'association Apronet, participent directement à la production et à la circulation d'un référentiel de priorités d'actions. Toutes ces instances vont ainsi se présenter comme des portes-paroles particulièrement actifs d'un nouveau modèle organisationnel pour les collectivités territoriales, modèle affirmant avec force le nécessaire passage d'une logique verticale à une logique transversale du management de l'information.

\section{Deux dispositions gouvernementales : coercitive et normative}

5 Résultant d'une convergence entre un impératif d'efficacité et le constat d'un déficit des administrations françaises en termes d'équipements TIC, les politiques gouvernementales créent un cadre institutionnel où les prescriptions des associations professionnelles (mais aussi de certains élus, des éditeurs de solutions technologiques...) vont trouver un écho inédit. En effet, deux dispositions institutionnelles vont particulièrement participer à l'accélération du déploiement des TIC. La première de ces dispositions relève d'une contrainte coercitive : il s'agit des politiques de modernisation des administrations françaises visant à en faire des " e-administrations. », et dont la promotion sera assurée dès 2003, par l'Agence pour le Développement de l'Administration Electronique (ADAE) et, à partir de 2006, par la Direction Générale de la Modernisation de l'Etat (DGME). Outre les politiques visant le développement des TIC, ces initiatives s'inscrivent dans la continuité d'une volonté gouvernementale de réforme de l'Etat initiée en 2001 et relative à l'optimisation de la gestion budgétaire publique (LOLF, La Loi Organique relatives aux Lois 
de Finances): ces nouvelles modalités de comptabilité s'appliquent à toute l'administration française depuis le $1^{\mathrm{er}}$ janvier 2006. Les maîtres-mots sont " performance », « transparence budgétaire », « management public », « responsabilités, « indicateurs ", « logique de résultat », " contrôle de gestion » et " audits ». Le modèle de l'entreprise privée est donc explicitement mobilisé. Contrainte normative, l'Agenda 21 est une seconde disposition issue des politiques étatiques en matière de Développement Durable. A la fin 2008, plus de 500 collectivités françaises l'avaient adopté. Les différents domaines de l'action politique locale étant concernés (le social, l'écologie, l'économie), l'atteinte des objectifs implique la coopération de directions différentes. Le référentiel de l'Agenda 21 indique la nécessité «de nouveaux modes de construction de la décision. [] Le développement durable sonne le glas d'une culture technocratique et d'un pouvoir hiérarchisé et repose sur un partage des savoirs, des pouvoirs et des responsabilités.» La transversalité devient ainsi une des conditions requises pour l'application effective de l'Agenda et le leitmotiv des nouvelles ingénieries d'info-communication.

\section{La traduction du couplage Intranet / Transversalité dans un Conseil Général}

\section{La recherche d'un nouveau positionnement intranet légitimé par l'Agenda 21}

6 Exerçant ses compétences sur un territoire départemental, un Conseil Général est composé d'une assemblée délibérante et d'un pouvoir exécutif ainsi que d'une organisation administrative ayant en charge la mise en œuvre des politiques votées et l'exercice de compétences spécifiques. Sous l'autorité du Président (élu), le directeur de l'administration (Directeur Général des Services) conçoit et décide les politiques et objectifs de l'administration. Le caractère bicéphale, le Politique et l'Administration, d'une telle organisation est évidemment à prendre en compte. Il convient aussi de souligner que, si l'intranet est consultable par les élus, il s'avère que dans le cas qui nous préoccupe, celui-ci ne reste qu'occasionnellement consulté par une minorité des conseillers généraux. Le projet d'évolution des processus de gestion de l'information concerne exclusivement l'administration et les différents agents qui travaillent dans ses directions. L'intranet a été créé en 1998 et, en 2008, il est déployé auprès de 2655 agents (sur les 3053 agents que compte le CG). Des accès en «libre service» sont également proposés aux agents des collèges. Relevant auparavant de la direction des ressources humaines, à partir de 2007, l'intranet dépend d'un service rattaché directement à la Direction Générale des Services. Il est doté de compétences existantes en son sein en matière de gestion de sites web et de la documentation. Mais globalement, on a encore une vision relativement restreinte de l'intranet auquel est attribuée essentiellement une mission de diffusion interne de l'information générale (discours du Président et du DGS en page d'accueil, informations sur les grands projets, de news générales, brèves - « l'info en continu du (G »-), et aussi, une mission de mise à disposition de procédures et de documents généraux (espace Presse \& Doc). Des rubriques dédiées à la présentation de chaque direction, un annuaire interne ainsi qu'un système d'information géographique complètent le dispositif. Adopté en 2006, le programme Agenda 21 décline un ensemble d'actions devant être menées d'ici à 2009, en posant notamment l'objectif d'une amélioration du service public. Associé à cet enjeu, «le projet d'évolution de l'intranet doit 
permettre en premier lieu, de renforcer la transversalité». "D'un outil purement informatif en 1998, l'intranet devient un outil de management, avec intégration des objectifs liés à la politique de développement durable».

\section{Le correspondant info : l'acteur désigné de la transversalité}

7 A la poursuite de son nouveau modèle organisationnel et info-communicationnel, le Conseil Général décide fin 2007, la mise en oeuvre d'un dispositif caractéristique des politiques de délégation des activités éditoriales dans les intranets " portail »: le réseau de contributeurs, ici dénommé réseau des « correspondants infos ». Ce type de dispositif constitue aujourd'hui une norme de pratiques : ainsi, selon l'étude 2008 de l'association Apronet, $82 \%$ des collectivités se seraient dotées d'un réseau de contributeurs.

8 L'idée est soutenue par le plus haut niveau de l'organisation: le Président du Conseil Général souligne, lors d'une réunion collégiale des acteurs intranets de novembre 2008, que "la création des correspondants info répond à une demande (que j'ai exprimée à plusieurs reprises), en cohérence avec l'esprit de l'agenda 21 ». Par ailleurs, le directeur général des services établit un lien permanent entre l'enrichissement de l'intranet, l'extension de la numérisation des documents et la nécessité de se donner des indicateurs permettant une évaluation continue des politiques publiques. Le poids de l'Agenda 21 dans la transformation à venir de l'Intranet est donc affirmé avec force et le nouveau modèle managérial, reposant sur la triade coopération/évaluation/transversalité, invoqué de manière soutenue. Il est brandi comme référence incontournable à une efficacité et une performativité de niveau supérieur à l'existant. Pour le dirigeant de l'administration, la délégation éditoriale est alors ce qui « doit donner chair » à la visée transversale, elle est la traduction de cet impératif. Le réseau se compose d'agents issus de directions différentes. Deux missions leur sont assignées : d'une part, la veille et la collecte d'informations relatives à l'actualité de leurs services; et d'autre part, la rédaction et la publication d'informations et de documents (ceux-ci pouvant aussi être exploités par le site internet). Certains agents ne traitent que la fonction de veille et n'assurent pas la rédaction. La présentation de la mission en précise l'intérêt et l'objectif pour les agents concernés : "Etre un vecteur d'informations; Favoriser les échanges transversaux et la circulation de l'information; Valoriser les actions de la direction et le travail des agents; Rendre l'actualité interne plus attractive, transversale, et concrète; Etre au ccur de l'information. Cela donne une place différente au sein de la direction. Un changement dans les missions quotidiennes, une ouverture sur la nouveauté, sur d'autres problématiques. Etre formé à de nouveaux outils. » Un responsable intranet assure l'animation, le suivi, l'assistance et le développement du réseau, ceci correspondant à près de la moitié de son temps de travail total. Les modes de désignation sont très variables et soumis à la décision du directeur du service : les correspondants-info occupent des postes très hétérogènes allant de l'assistante de direction d'un service jusqu'au manager. A fin 2008, le réseau des correspondants-info et des veilleurs compte une soixantaine de personnes, dispersées sur les différents sites de la collectivité.

9 L' évolution technologique a également été saisie comme une opportunité pour la mise en oeuvre d'une délégation des tâches auparavant dévolues au webmaster intranet. Ayant principalement construit leurs standards fonctionnels à partir des projets vendus aux entreprises privées, les solutions intranets, dites "portail», se sont imposées aux collectivités (l'offre se caractérisant par l'idée d'outils de publication « intuitifs » et une 
étiquette collaborative). La solution Sharepoint, utilisée par le Conseil Général, est un produit phare de l'offre Microsoft en matière d'intranet. L'éditeur la positionne explicitement dans sa contribution à la transversalité : «SharePoint relie le collaborateur et son environnement de travail. Pour contrôler et piloter l'activité collaborative transversale, les processus génériques de SharePoint pourront être complétés par les processus métier verticaux d'une application de gestion de l'information." Ici, à l'instar de nombreuses autres organisations, la rencontre entre l'objet technique et le projet politique constitue un levier et une justification supplémentaire au projet. En offrant une interface a priori utilisable par des néophytes de l'administration web, ces plates-formes laissent entrevoir un partage facilité de l'activité éditoriale entre la direction de la communication et un réseau d'acteurs susceptibles, eux aussi, de produire du contenu.

\section{L'évaluation du lien intranet - transversalité : entre enthousiasme du management et perplexité des intranautes}

Pour les responsables managériaux, efficacité de l'intranet et transversalité renvoient à six thématiques principales : l'affranchissement des intermédiaires ( $s^{\prime}$ 'affranchir des secrétaires »); la circulation de l'information ("l'accès étendu à l'information »); une nouvelle «sociabilité» au sein de l'organisation («l'intranet doit être une incitation à aller vers les autres »); l'enrichissement des points de vue («il doit favoriser une interaction entre directions et agents »); une meilleure connaissance/visibilité des activités et missions des services (« les correspondants info permettent de voir ce qui se passe à l'intérieur d'un service»). Enfin, certains managers n'hésitent pas à associer le réseau de contributeurs à une «démarche de démocratie participative ». Mais, au delà des légitimations politiques, on constatera la faiblesse de la traduction effective de certaines promesses et visées ainsi énoncées. De plus, alors que l'exigence d'indicateurs et d'évaluation est rappelée sans cesse par le directeur général, les statistiques de consultation de l'intranet sont inexistantes. Après plus d'un an d'existence, la collectivité va réaliser un bilan de l'expérimentation «correspondants-info ». Les indicateurs privilégiés visent à «matérialiser » et objectiver le dispositif et ses gains. Mais, seuls sont retenus des critères pour le moins éloignés d'une réflexion sur les nouvelles activités coopératives et la circulation des savoirs. Sont considérés : le temps passé à la mission de correspondant-info et/ou veilleur; le temps passé en formation; le niveau de contribution (identification des forts et faibles contributeurs); et au final, une information quantitative sur le nombre de pages intranets ou de rubriques créés via le réseau. Si au niveau de la direction politique et administrative, le satisfecit est général, du côté des agents, si le projet suscite un intérêt, il est aussi marqué d'un fort scepticisme. Ainsi : l'accès et l'utilisation d'une information à vocation transverse repose sur des modes de filtrage et de circulation qu'ils ne comprennent ou ne connaissent pas spontanément ; la question d'une appropriation de l'intranet renvoie de manière forte, à un rapport instable de substitution / complémentarité entre les réseaux sociaux dit « réels » et les TIC. De ce point de vue, les dimensions « humaines, affectives et conviviales » du procès d'information, à travers la culture de ce que certains agents nomment la culture de la «brève de comptoir » ou « machine à café ", sont largement rappelées. Le lien entre Intranet, réseau de contributeurs et transversalité est donc loin d'être explicite pour les utilisateurs de l'intranet, d'autant plus que les politiques documentaires qui perdurent s'avèrent en contradiction avec l'affichage d'un projet politique axé sur la collaboration inter-services. Ainsi, l'utilisation des serveurs de fichiers indique comment la mise en pratique d'une mutualisation reste 
problématique. Ces serveurs sont organisés selon leurs taxonomies conçues (de manière très complexe) localement par une direction ou un service, s'accompagnent de droits de consultation restreints, et n'offrent pas de fonctionnalité de recherche. Il en résulte un cloisonnement assuré au niveau d'un service qui maintient ainsi son territoire documentaire propre. Enfin, l'observation des pratiques intranautes montre comment chacun reste au plus près de ses centres de compétences et d'intérêts (son service, son équipe, son information personnelle, notamment relative aux sujets ressources humaines). Selon un contributeur, l'architecture de l'intranet ne favorise pas une exploration des territoires informationnels : "en dehors des rubriques de mon service, ça me rebute d'aller sur intranet, car je ne sais pas comment accéder aux infos d'autres services ». Pour un autre : " on arrive à aller aux informations que l'on connaît en rapport à son service, mais en dehors de lui, je ne connais rien, ou quand j'ai essayé d'aller ailleurs dans l'intranet, j'ai trouvé cela trop compliqué. Et si jamais je trouve une information intéressante d'un autre service, je ne sais plus après quel est le chemin que j'ai pris pour y arriver». De même, les résultats de l'enquête quantitative montrent que l'apport de l'intranet à une plus grande coopération entre services, à un décloisonnement ou au partage des savoirs, est encore perçue de manière toute relative. L'acquis en la matière est principalement assigné à la diffusion des actualités mises en ligne par les contributeurs en page d'accueil (selon le service communication interne, "la sensibilisation à la transversalité se fait par les événements"). Donc, en dehors de ce type d'informations éphémères, les gains en termes de transversalité sont peu perçus : seulement $27 \%$ considèrent que l'intranet permet de mieux partager les savoirs, $75 \%$ disent ne pas avoir connaissance ou un accès facile aux documents produits par d'autres services que le leur, et enfin, le collègue ou la hiérarchie restent de loin, les sources premières d'acquisition d'un document. La persistance du modèle structurel et bureaucratique en matière de management de l'information est constatée par une majorité d'agents et l'architecture d'information de l'intranet ne rompt pas avec le modèle hérité.

11 Néanmoins, la demande de création d'espaces collaboratifs (dédiés à la gestion des projets et inexistants pour l'instant) se présente alors comme la trace la plus explicite d'un besoin de facilitation des coopérations transversales de groupes projets. En l'absence de telles solutions technologiques, la messagerie maintient son hégémonie en tant qu'outil privilégié de partage des ressources.

\section{Nouvelle gouvernance éditoriale et sémio-politique : l'expression de tensions associées aux activités de contribution}

\section{Problème d'encadrement et de reconnaissance de l'activité de contribution}

12 La délégation éditoriale s'accompagne couramment de la création d'une charte éditoriale. C'est un outil d'encadrement des contributions décentralisées (ou des créations de sites, de rubriques etc.) qui pose donc différentes normes portant essentiellement sur le respect de la forme (charte graphique, navigation, formats des documents, écriture écran etc.); sur les axes éditoriaux (thématiques traitées par les responsables de rubriques); sur le processus de publication définissant le niveau d'autonomie du contributeur. Donc, la 
gouvernance éditoriale maintient des routines de contrôle ou les transforme, et le réseau de contributeurs se présente alors comme un compromis entre une expression distribuée et des désirs persistants de centralisation. Dans le Conseil Général, le droit formel à l'expression et à la contribution s'exprime de manière hétérogène en fonction des services : dans des fiches de postes pour certains agents contributeurs ou veilleurs ; par une obligation ou pas de validation amont du hiérarchique. Mais, alors que le rôle de correspondant-info est vécu comme une valorisation, les contributeurs interrogés soulignent ici l'absence de normes et de principes communs qui devraient définir leur statut. Ils insistent sur l'existence de modes de contrôle différenciés, et ceux qui seraient soumis à une validation préalable de leur production, expriment la subjectivité d'un dispositif fondamentalement soumis au «bon vouloir» du responsable de service, à un processus qui, souvent, "n'est pas nécessaire ». Une hiérarchie entre correspondant-info se dessine: les autonomes et les contrôlés; les correspondants reconnus statutairement (détenteurs d'une fiche de poste enrichie de la mission) et les correspondants non institués. Se révèle ainsi le caractère paradoxal de la politique menée articulant d'une part, une exigence de production croissante d'information, une délégation de l'activité éditoriale impliquant autonomie et initiative, et d'autre part, la perpétuation de modes de contrôle traditionnels. Pour la direction, l'absence de normes communes quant au processus de validation s'impose quand il s'agit de négocier localement et de convaincre chaque responsable de la pertinence du dispositif. Selon elle, il convient dans certains cas de rassurer et «maintenir le chef de service dans son rôle symbolique de décideur final ».

\section{L'expression d'un désir de cadrage et de professionnalisation}

13 Pour un manager, le correspondant-info doit «veiller à tous les étages de la DG », et pour un autre, « tous les collègues sont des veilleurs ». Ainsi, l'ensemble de l'organisation deviendrait un vivier de ressources informationnelles que l'agent devrait capter et suivre. Or, sa réalité professionnelle s'accorde peu avec ces prescriptions. Le cloisonnement encore massivement perceptible entre directions et les objectifs immédiats de l'agent n'incitent pas à étendre les territoires de veille. Les veilleurs rappellent ainsi que si le développement d'un réseau social proche et riche est une condition de réussite de la mission, cela requiert un temps disponible qu'ils ne possèdent pas: «j’ai déjà du mal à connaître les collègues de ma direction et les différentes actions de mon service, alors je ne peux pas aller ailleurs pour savoir ce qui se passe». En l'absence de transmission spontanée d'informations à leur intention, ils sont donc soumis à un processus d'acquisition de l'information fastidieux. Le deuxième axe de la mission, à savoir le filtrage d'informations, la production et la mise en ligne de contenus est également débattu. En conservant son poste et ses activités professionnelles initiales, le correspondant-info se présente comme un hybride se déplaçant sur des zones de missions allouées traditionnellement au communicant et les objectifs éditoriaux varient selon les directions : le correspondant info est soit pensé comme opportunité de mise en visibilité et de valorisation auprès de toute la collectivité des actions d'une direction ( l'objectif est de " Se faire voir et se faire connaitre auprès $d u(G »)$, soit comme un levier de communication entre les équipes de sa direction ( « se connaître entre nous », " faire lien » entre agents d'une même direction). On assiste donc à une diversité d'incarnation de la mission : certains correspondants-info vont concevoir leurs pages comme un espace dédié avant tout à leur direction et penser le territoire numérique dont ils ont la charge comme un espace collaboratif (l'axe de publicisation auprès d'autres directions est mis en mineur et aucune 
considération particulière n'est portée aux problématiques de langages spécialisés). D'autres, vont essentiellement traiter le volet "présentation générale», le volet événementiel et les actualités de leurs directions, en se centrant alors ici sur la production d'infos en page d'accueil de l'intranet. Les derniers mixent les deux positionnements, en créant deux espaces et deux modes de navigation. En l'absence d'un cadrage clair de sa direction, le correspondant info traduit et « actualise » ainsi sa mission en fonction de ses perceptions et visées personnelles, cadrage dont certains expriment la nécessité, au même titre qu'un retour sur l'utilité de leurs actions (revendication de statistiques de consultation, totalement absentes aujourd'hui). Cela est l'indice d'un désir de professionnalisation, d'une reconnaissance des compétences infocommunicationnelles qu'ils ont développées, et plus globalement, de sens pragmatique donné à leurs missions, qui s'avère ici en grande partie évacué.

\section{L'écriture des « territoires informationnels " ou l'enjeu politique attaché à une délégation du pilotage sémiotique de l'organisation}

14 Les sémiotiques (ergonomies, architectures d'information, modes de navigation, politiques éditoriales) se présentent comme l'expression «sensible» de ce compromis instable entre l'organique, le hiérarchisé et les micro-localités diffuses, entre une structuration normée et les espaces éditoriaux plus ou moins autonomes, émergents. A travers son régime d'interfaces, l'intranet relève d'une esthétique organisationnelle qui, loin d'être une expression strictement graphique ou métaphorique de l'organisation, engage l'analyse du couplage socio-technique dans ses dimensions sémio-politiques. Dans le prolongement de travaux réalisés sur ces questions (Gryspeerdt, Marion, 2002), la figure socio-esthétique de l'intranet renvoie encore à une carte socio-cognitive de l'organisation, carte et modélisation mouvante dont l'affordance (Gibson, 1977) est soumise aux dialectiques se déployant au niveau d'une ingénierie des activités éditoriales, et ce, bien au-delà d'un design essentialisé par la seule question des marqueurs identitaires. Le script TIC-Transversalité nous amène ainsi à examiner, à partir des dispositifs éditoriaux distribués et des contraintes-tensions qui les traversent, ce que nous pouvons définir plus globalement, après F.Guattari et E.Alliez, comme la fondation d'une politique des moyens de pilotage sémiotique (Guattari, Alliez,1983).

Si on s'en tient au plan local de cette organisation, ces dimensions sémio-politiques se développent particulièrement sur deux axes. Le premier, inhérent à la délégation éditoriale et à cette économie de la contribution, met en exergue l'enjeu d'une occupation de l'espace de la « home». Les responsables de directions ainsi que leur correspondants info respectifs ont compris les nouvelles potentialités qui leurs sont offertes via l'autorisation de publication directe d'informations sur cette page (seules les "actus " peuvent y être publiées, et ce, sans filtrage systématisé du responsable intranet). Ainsi, afin «d'occuper l'espace", certaines directions vont exiger de leur contributeur, une production fréquente de brèves d'actualité. Si certaines informations relèvent de besoins opérationnels ou transversaux (par exemple, informer d'un retard sur le versement de la paye -cas exceptionnel-, ou d'un incendie en centre ville), l'objectif visé est d'être vu en permanence. Dans cet investissement de la page d'accueil, les correspondants-infos voient une opportunité de valorisation du service mais aussi de valorisation personnelle. Etre auteur d'une info en page d'accueil semble fortement chargé d'une puissance symbolique et la compétition entre correspondants, clairement exprimée. En effet, 
l'affichage étant limité à six brèves de directions par jour, il est nécessaire d'en créer de nouvelles régulièrement pour espérer être vu. Le deuxième axe de débats sur la traduction sémiotique de l'organisation est exprimé en majeur par les responsables managériaux. Se trouve ici à nouveau questionnée l'incarnation de la transversalité dans l'architecture d'information. Est alors posée la nécessité d'accéder à des informations qui pourraient se classer, non pas selon une logique structurelle, mais selon une "matrice territorialiste ", une logique de "coopération territoriale ", plutôt qu'une vision "DGA " ou (en écho aux entreprises privées) une vision "filiales, activités ". "Sur un territoire dont le filtre pourrait être une ville, un canton etc., il conviendrait d'avoir une vision des directions, services, personnes, projets impliqués, mais aussi des élus concernés ainsi que des partenaires. » Or, tous n'adhèrent pas à l'idée d'une visibilité augmentée de ses ressources informationnelles et de ses activités. Le partage de l'information comme nouveau paradigme culturel est à nouveau convoqué et s'accompagne alors d'une rhétorique usitée, encore mobilisée par le président du Conseil Général : "Ceux qui persistent à croire que conserver l'information donne du pouvoir, n'ont rien compris!». Et, comme le souligne le directeur général, «appliquer la transversalité implique que les directions acceptent que l'on puisse regarder chez elles, et c'est loin d'être le cas.» Un enjeu majeur semble ici effacé des débats, alors qu'il est, à notre sens, constitutif des inerties organisationnelles: le caractère bicéphale d'une collectivité et donc la question des informations attachées aux élus. Seules quelques réflexions, une fois encore émanant d'acteurs contributeurs ou d'intranautes, soulignent l'accès problématique aux informations relevant de l'action politique, celles-ci impliquant pour les directions une recherche difficile de divers documents. Un responsable intranet d'une autre collectivité nous confiait récemment les inquiétudes de sa direction générale par rapport aux potentialités d'un nouveau moteur de recherche qui permettrait un repérage plus efficace de l'information: "Ne risque t-on pas de mettre en visibilité l'absence d'équité entre les actions menées sur un territoire? Comment pourrait -on maintenir un privilège d'information par rapport aux élus de l'opposition qui peuvent eux aussi utiliser l'intranet ? Faut-il risquer de perdre un avantage concurrentiel sur l'information?" Un nouveau moteur de recherche, capable de mettre en visibilité et d'associer les mondes informationnels d'un élu, pourrait-il être perçu comme un outil d'évaluation "par destination » de l'action politique? Là encore, on voit bien que les scripts socio-techniques mettent en tension l'injonction à la transversalité et qu'ils sont au cœur d'une négociation politique de la transparence et de l'opacité informationnelle.

\section{Conclusion}

Analyser les manières dont résonnent, de façon tantôt convergente et tantôt divergente, les politiques organisationnelles et leurs dispositifs idéels et matériels dédiés à la performation stratégique et tactique des processus de travail, ainsi que les micropratiques avec leurs propres narrations, permet d'observer la manière dont les salariés, en cherchant tout à la fois à adopter les nouveaux dispositifs, ne cessent aussi d'introduire altération et création vis-à-vis des scripts managériaux et techniques. A partir de notre terrain, nous avons tenté, dans une optique essentiellement constructiviste, de saisir un certain nombre de dimensions et difficultés attachées à la coexistence complexe de narrations aux statuts très divers. Ont été posés quelques repères pour une approche critique de ce champ narratif non exclusivement linguistique, champ par lequel et à travers lequel se joue l'actualisation des pratiques professionnelles, des 
nouvelles formes organisationnelles, leur performation. De même, nous avons essayé de proposer quelques appuis permettant d'entrevoir ce que pourrait être une polémologie narrative, c'est-à-dire, une narratique incluant les sémiotiques socio-techniques et convoquant une vison pragmatique des récits, de leurs entrelacs et /ou de leur relative autonomie. Mise en acceptabilité et adoption des TIC (Stiegler, 1996) sont au cœur de cela. L'examen des pratiques info-communicationnelles ne saurait passer outre cette narratique générale (Latour, 2006; Faye, 1972), celle-ci devant prendre sur elle, l'appréhension des agencements collectifs d'énonciation et les équipements collectifs de subjectivation (Deleuze, Guattari, 1980), leurs combinaisons plus ou moins complexes, qui servent de milieux aux processus de construction polémiques, politiques, de l'eorganisation.

En développant de tels axes, nous pensons pouvoir mieux appréhender les pragmatiques internes à l'œuvre dans les collectifs de travail, ne serait-ce que par leurs usages quotidiens des TIC et par les ajustements, les bricolages, qu'ils opèrent, et ouvrir ainsi, à une meilleure perception des processus de subjectivation qui se trouvent à la traversée des rhétoriques technologiques, des stratégies discursives hétérogènes des différents acteurs. Dans ce cadre, notre approche qui prend pour actant pivot les scripts, avec leurs sémiotiques et leurs régimes de fonctionnement très divers, nous semble devoir être approfondie, jusqu'à développer une "polémologie des sémiotiques» traversant les couplages socio-techniques. Ce que nous visons, c'est l'élaboration progressive d'une sémio-politique de l'innovation. impliquant acteurs humains et non-humains.

\section{BIBLIOGRAPHIE}

Akrich, M., (1987), « Comment décrire les objets techniques ? », in Techniques et culture, $n^{\circ} 9, \mathrm{pp}$ 49-64.

Boutang, YO., (dir) (2007), Le capitalisme cognitif: La nouvelle grande transformation, Paris : Eds Amsterdam.

Callon, M. et Latour, B., (1981), « Le grand Leviathan s'apprivoise t-il ? »; in Akrich M.

Callon, M., Latour, B., (2006) ; Sociologie de la traduction: textes fondateurs ; Les Presses - Mines Paris (1ère publication 1981 : "Unscrewing the Big Leviathan; or How Actors Macrostructure Reality, and How Sociologists Help Them To Do So?" in Karin D. Knorr, and Aron Cicourel (dir.) Advances in Social Theory and Methodology. Toward an Integration of Micro and Macro Sociologies, London: Routledge \& Kegan Paul, pp. 277-303).

Callon, M. et Latour, B., (1991), La science telle qu'elle se fait, Paris : La Découverte.

Carmes, M., (2008-a), « Proposition pour une analyse critique de l'innovation organisationnelle : le cas de l'e-organisation et de ses associations professionnelles», Colloque international EUTIC 08, Au carrefour des mondes numériques, Faculdade de Ciências Sociais e Humanas , Lisbonne, 22/25 octobre, Actes (à paraître), 13 pages. 
Carmes, M., (2008-b), «L'organisation peut-elle s'inventer par les scripts ? Performations et actualisations dans les agencements Intranet ", Actes du colloque Les dispositifs de médiation organisationnelle, technologique et symbolique dans la communication des organisations, Laboratoire I3M Université de Nice Sophia Antipolis, 4-5 décembre, pp 260-268.

Deleuze, G. et Guattari, F., (1980), Mille plateaux, Paris : Les Editions de Minuit.

Faye, JP., (1972), Théorie du récit : introduction aux "Langages Totalitaires", Introduction à une narratique générale, Edition Hermann.

Gibson, JJ., (1977), « The Theory of Affordances and the environment's design », Communication présentée au symposium La perception en architecture, Toronto, partie VIII .

Guattari, F., Alliez, E., (1983), « Le Capital en fin de compte : systèmes, structures et processus capitalistiques», in Revue Change International, Travail/Energie : la crise et l'apocalypse, Numéro 1.

Gryspeerdt, A., et Marion, P. (coord) (2002), Esthétique des organisations, Revue Recherches en Communication, $\mathrm{n}^{\circ} 17$.

Latour, B., (2006), Changer la société, Refaire de la sociologie, Paris : La découverte

North, D., (1990), Institutions, Institutional Change and Economic Performance, Cambridge, Cambridge University Press, in Chavance, B. (2007), L'Economie Institutionnelle, La Découverte.

Powell, W. et Di Maggio, PJ., (1991), The new institutionnalism in organizationnal analysis, Chicago : The University of Chicago Press.

Stiegler, B., (1996), La technique et le temps, Tome 2 : La désorientation, Galilée.

\section{RÉSUMÉS}

A partir du déploiement de nouvelles gouvernances éditoriales, cet article interroge un agencement qui participe au formatage des politiques organisationnelles, ainsi que les processus normatifs et coercitifs qui le caractérisent. En amont, il repose la question des rapports entre des pratiques locales et un mot d'ordre, entre une injonction gouvernementale portant sur l'eadministration et son application au sein des ingénieries info-communicationnelles rattachées aux intranets. Les nouvelles politiques d'écritures et de contribution décentralisée, leur référentiel d'actions, sont envisagés comme un "script " issu du couplage intranettransversalité. On examine alors le dispositif d'un Conseil Général, les discours des chefs de projets, la place des objets techniques, les pratiques et perceptions du réseau de contributeurs, ce dernier étant l'incarnation majeure d'une mise en conformité de l'organisation vis-à-vis de l'exigence de transversalité. Cette recherche montre donc la manière dont l'activité de contribution se trouve être, à la fois, aux prises avec l'intranet et avec l'injonction à la transversalité, ainsi qu'une dichotomie entre la volonté politique affichée et l'application hétérogène des visées politiques et stratégiques. Des tensions s'expriment ici massivement sur les modes de régulation et les objectifs de l'activité de contribution, sur la reconnaissance et le développement de nouveaux professionnalismes info-communicationnels, sur l'investissement de l'interface et la mise en visibilité des territoires. Ce faisant, on propose l'élaboration progressive d'une sémio-politique de l'innovation organisationnelle à partir d'une combinatoire complexe des narrations.

From the analysis of new ways developped by the publishing management, within the intranets, the purpose of this article is to offer a framework to understand the « socio-technical collective assemblages " that shape the praxis and the politics of new organizations. Besides, these « 
collective assemblages " shape the whole coercive and prescriptive processes. We closely observe, the relations between a governmental injunction concerned of the digital administration developement and the organizational cooperations. We study the translation process of this injunction (in the way of Callon - Latour) through the implementation of information and communication machinery. More precisely, the machinery embodied in the collaborative intranets.

The " new economy of contribution » and its system of reference are considered as operators of transversality or, " transversality script ". Next, we explore in a pragmatical way the organization of a "Conseil Général " and we examine the intranet praxis through the perceptions of a specific network of « correspondants-infos ». This terrain allows us to underline the great gap between the political views expressed by the compagny managers and hox transversality is actually carried out. It is the scene of political and organizationnal conflicts and controversies. These sophisticated implementations of pilotage, these politics of semiotics cause hard controversies about the new regimes of visibility inside the digital territories.

\section{INDEX}

Mots-clés : administration, collectivités territoriales, contribution, intranet, transversalité, sémio-politique, TIC, management de l'information, organisation

Keywords : administration, intranet, local authorities, transversality, contribution, semiopolitic, ICT, information management, organization

\section{AUTEUR}

\section{MARYSE CARMES}

Maryse Carmes est docteur en Sciences de l'Information et de la Communication ( Unité de Recherche « LABORATOIRE PREFICS » (UMR CNRS 8143). « Equipe disciplinaire» CERSICUniversité de Rennes 2 ) et réalise des travaux de recherche portant sur une analyse des politiques TIC en organisation et des ingénieries d'info-communication. Elle développe une approche critique des dynamiques d'innovation et plus globalement, étudie la formation et les évolutions d'un capitalisme cognitif sous ses diverses formes et traductions. Elle enseigne à l'université de Rennes II et à l'Université Catholique de l'Ouest. 